\title{
SELF-ABSORPTION ANALYSIS OF SILVER RESONANCE LINES IN NANO-MATERIAL LASER PRODUCED PLASMA
}

\author{
ASHRAF M. EL SHERBINI ${ }^{1}$, MOHAMED A. HAGRAS ${ }^{2}$, MOHAMED R. RIZK ${ }^{2}$, \\ EL-SAYED A. EL BADAWY ${ }^{2}$, CHRISTIAN G. PARIGGER ${ }^{3 *}$ \\ ${ }^{1}$ Laboratory of Laser and New Materials, Faculty of Science, \\ Cairo University, Giza, Egypt \\ ${ }^{2}$ Faculty of Engineering, EE Department, Alexandria University, Egypt \\ ${ }^{3}$ University of Tennessee Space Institute, Center for Laser Applications, \\ 411 B.H. Goethert Parkway, Tullahoma, TN 37388-9700, USA \\ *Corresponding author E-mail: cparigge@ tennessee.edu (C.G. Parigger)
}

\begin{abstract}
The resonance spectra of neutral silver indicate self-absorption for the studied Ag I lines at the wavelengths of $327.9 \mathrm{~nm}$ and $338.2 \mathrm{~nm}$. The center dip is associated with self-reversal due to self-absorption in the plasma. The Q-switched radiation of $355 \mathrm{~nm}, 532 \mathrm{~nm}$, or $1064 \mathrm{~nm}$ from a Nd:YAG laser device generates the plasma at the surface of silver nano-material targets, with experiments conducted in standard ambient temperature and pressure laboratory air. Procedures for recovery of the spectral line shapes confirm that over and above the effects of selfreversal, line shape distortion are important in the analysis. The work discusses parameters describing selfabsorption when using fluence levels of 2 to $33 \mathrm{~J} / \mathrm{cm}^{2}$ to generate the plasma. Furthermore, subsidiary calibration efforts that utilize the hydrogen alpha line of the Balmer series show that the Ag I lines at $827.35 \mathrm{~nm}$ and $768.7 \mathrm{~nm}$ are optically thin.
\end{abstract}

Keywords: Laser-induced plasma; atomic spectroscopy; self-absorption; nanoparticles; silver; hydrogen;

\section{INTRODUCTION}

Self-absorption of radiation from plasma can occur due to re-absorption in a cooler region. For instance, line-ofsight measurements of laser-induced plasma at or near an ice surface ${ }^{1}$ show self-reversal tips at the un-shifted resonance wavelength of the hydrogen alpha line of the Balmer series. Typical "fingerprints" due to re-absorption include self-reversal and line-shape distortions ${ }^{2-8}$. In this work, two parameters distinguish between these effects.

There are significant challenges when considering self-reversed lines for evaluation of the electron density typically measured from the full-width-at-half-maximum, and determination of the temperature that is a function of the spectral radiance. Moreover, spectral line intensities from nano-based materials show differences from the corresponding bulk signals ${ }^{9}$. The theoretical description of self-absorption ${ }^{10,11}$ relies on the computation of the emitted radiation when modeling the emitters by a specific distribution.

\section{EXPERIMENTAL DETAILS}

In the experiments, a Nd:YAG laser device(Quantel model Brilliant B) operates at the fundamental wavelength of $1064 \mathrm{~nm}$ and the two harmonics at 352 and $355 \mathrm{~nm}$, with output laser energy per pules of $370 \pm 5,100 \pm 4$, and $30 \pm 3 \mathrm{~mJ}$, respectively. The corresponding spot sizes at the target surface amount to $0.5 \pm 0.05,0.44 \pm 0.05$, and $0.27 \pm 0.03 \mathrm{~mm}$. An optical fiber of $400 \mathrm{~mm}$ diameter collects the radiation from the plasma. A SE200 Echelle type spectrograph with an average instrumental bandwidth of $0.2 \mathrm{~nm}$, and an attached intensified chargecoupled device (Andor iStar DH734-18F) acquire the data. The spectral pixel resolution and pixel area amount to 
$0.02 \mathrm{~nm}$ and $196 \mu \mathrm{m}^{2}$, respectively. A xyz-holder allows one to position the optical fiber at distance of $5 \mathrm{~mm}$ from the laser-induced plasma. A $500 \mathrm{~kg} / \mathrm{cm}^{2}$ press prepared the silver nanomaterial powder (from $\mathrm{MKNANO}^{\circledR}$ ) to produce a less brittle tablet without further purification or heat treatments. The nanoparticle size equals $100 \pm 5 \mathrm{~nm}$, as confirmed from measurements with a transverse electron microscope.

The time delay and gate width amount to $2 \mu$ s for all experiments reported in this work. ICCD KestrelSpec ${ }^{\circledR}$ software subtracts the background stray light contributions. The measured electronic noise level amounts to $20 \pm 7$ counts across wavelength range of $250-850 \mathrm{~nm}$. The measurements of incident laser energy at each laser shot utilize a quartz beam splitter to direct the reflected part (4\%) to a calibrated power-meter (Ophier model 1z02165). A 25 ps fast response photodiode in conjunction with digital storage CRO (type Tektronix model TDS 1012) measures the laser pulse width of $5 \pm 1 \mathrm{~ns}$. A set of calibrated neutral density filters adjusts the energy/pulse. The DH2000-CAL lamp (Ocean Optics SN037990037) allowed us to correct for the sensitivity of detection system composed of spectrograph, intensified camera and optical fiber.

\section{RESULTS AND DISCUSSION}

For the resonance transitions of the $\mathrm{Ag}$ I lines at 327.9 and $338.2 \mathrm{~nm}$, Figures 1 to 3 illustrate recorded and fitted nano-material silver lines with central dips at line center. The Stark shift is smaller than the instrument width.

(A)

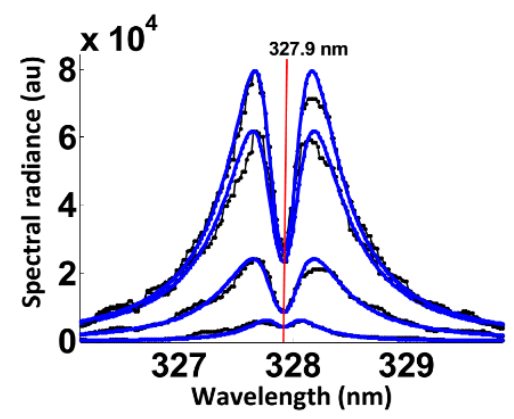

(B)

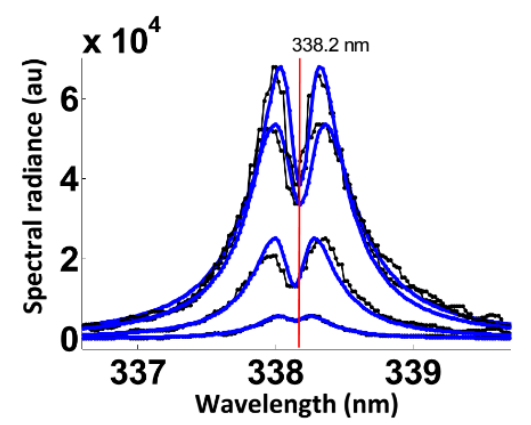

Figure 1: Self-reversal of AgI (A) $327.9 \mathrm{~nm}$ and (B) $338.2 \mathrm{~nm}$ lines; $355 \mathrm{~nm}$, fluences of 13.5, 9.6, 5 and $2.1 \mathrm{~J} / \mathrm{cm}^{2}$.

The two sets of spectra show the results following irradiation of nano-material silver targets with $355 \mathrm{~nm}$ radiation. The self-reversal of plasma radiation from nano-silver material is typically absent in investigations of laser-induced plasma with bulk-silver targets for otherwise similar experimental conditions. Figure 1 shows well-developed spectral dips. Accordingly, Fig. 2 displays recorded spectra obtained with 532 nm excitation.

(A)

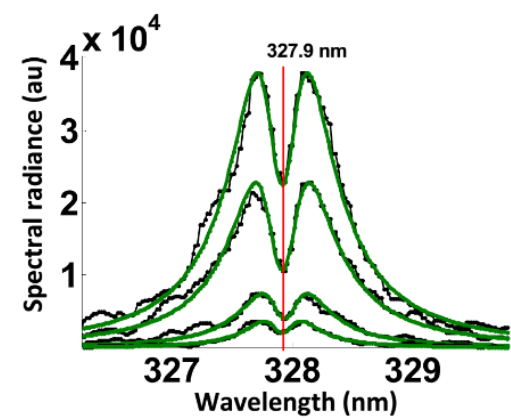

(B)

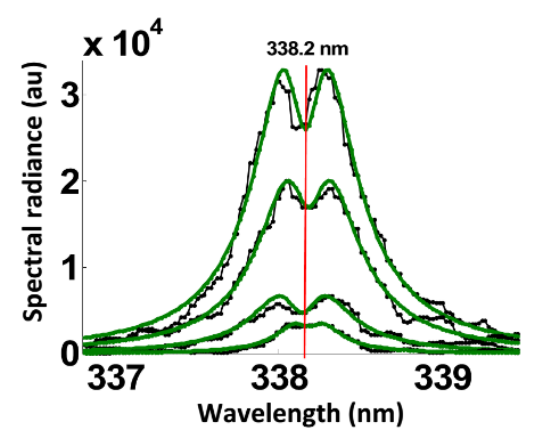

Figure 2: Self-reversal of AgI (A) $327.9 \mathrm{~nm}$ and (B) $338.2 \mathrm{~nm}$ lines; $532 \mathrm{~nm}$, fluences of 13.5, 11.5, 8, and $6 \mathrm{~J} / \mathrm{cm}^{2}$. 
Figure 2 indicates diminished self-absorption when compared to Fig. 1. For $1064 \mathrm{~nm}$ laser excitation, Fig. 3 indicates even smaller self-absorption phenomena for the two silver lines.

(A)

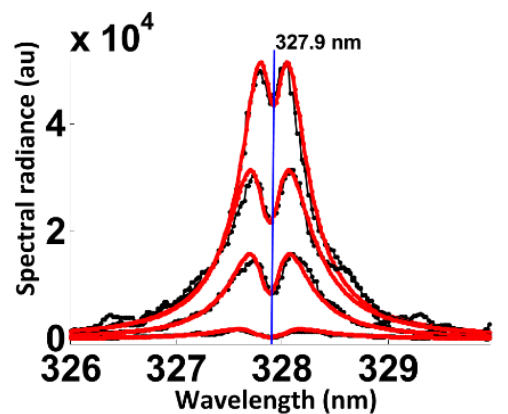

(B)

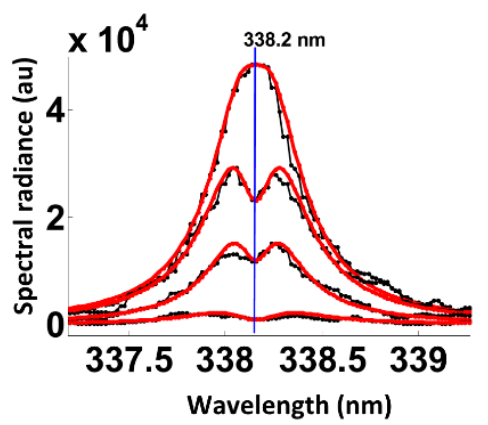

Figure 3: Self-reversal of AgI (A) $327.9 \mathrm{~nm}$ and (B) $338.2 \mathrm{~nm}$ lines; $1064 \mathrm{~nm}$, fluences of 13.5, 11.5, 8, and $6 \mathrm{~J} / \mathrm{cm}^{2}$.

In view of Figs. 1 to 3, one can see that it would be challenging to extract the full-width at half-maximum for determination of electron density. Instead, two Ag I lines at $827.35 \mathrm{~nm}$ or at $768.7 \mathrm{~nm}$ are suitable for electron density measurements due to absence of self-absorption. The inferred electron densities compare nicely with the corresponding values obtained from analysis of the hydrogen alpha line of the Balmer series. Figure 4 illustrates the results, and Table 1 shows the comparisons.

(A)

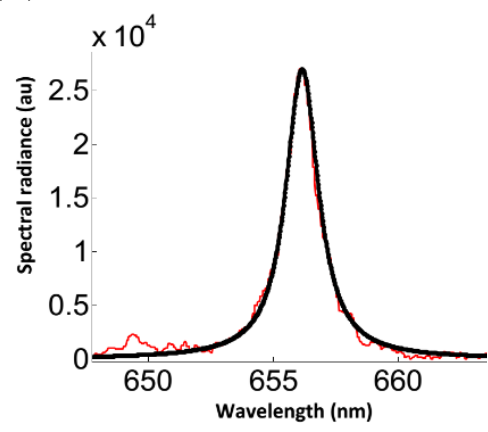

(B)

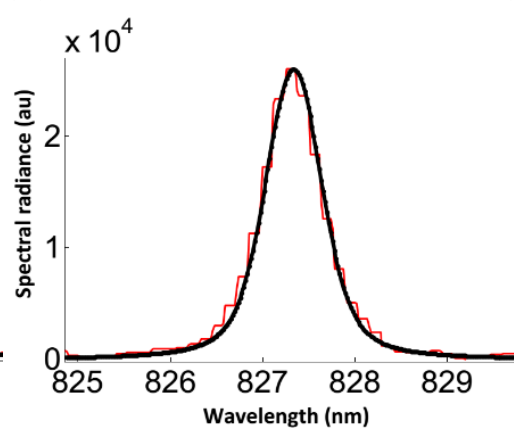

(C)

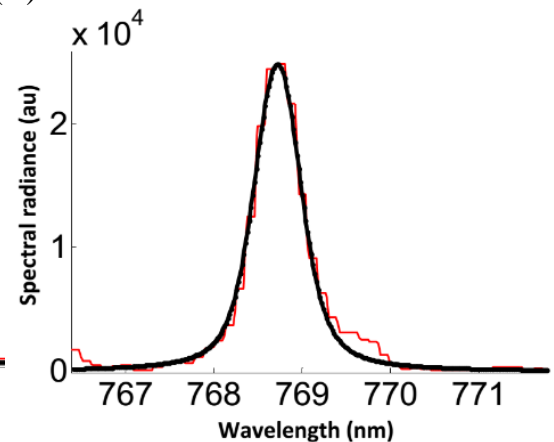

Figure 4: Recorded spectra for (A) $\mathrm{H}_{\alpha}$ at $656.28 \mathrm{~nm}$, (B) $\mathrm{Ag}$ I at $827.35 \mathrm{~nm}$, and (C) AG I at $768.7 \mathrm{~nm}$, for IR plasma excitation and for a fixed laser fluence of $9.6 \mathrm{~J} / \mathrm{cm}^{2}$.

Table 1: Electron densities, $\mathrm{n}_{\mathrm{e}}$. in units of $10^{17} \mathrm{~cm}^{-3}$ for different $1064 \mathrm{~nm}$ fluence.

\begin{tabular}{c|ccc}
\hline Laser fluence $\left(\mathrm{J} / \mathrm{cm}^{2}\right)$ & $\mathrm{n}_{\mathrm{e}}\left(\mathrm{H}_{\alpha}-656.28 \mathrm{~nm}\right)$ & $\mathrm{n}_{\mathrm{e}}(\mathrm{Ag} \mathrm{I}-827.35 \mathrm{~nm})$ & $\mathrm{n}_{\mathrm{e}}(\mathrm{Ag} \mathrm{I}-768.7 \mathrm{~nm})$ \\
\hline 9.94 & 1.64 & 1.66 & 1.76 \\
7.46 & 0.76 & 0.77 & 0.76 \\
5.9 & 0.63 & 0.66 & 0.7 \\
4.47 & 0.57 & 0.55 & 0.58 \\
\hline
\end{tabular}

For analysis of the self-absorbed spectra in Figure 1, notice the line reversal at the center wavelength, $\lambda_{0}$, and weaker effects in the wings leading to distortions. The transmittance, $T\left(\tau_{\lambda_{0}}\right)$, is related to the escape factor of the plasma $^{10,11}$, and it depends on the optical thickness of the plasma, $\tau_{\lambda_{0}}$. The transmittance ${ }^{2,3}$,

$$
\mathrm{T}\left(\tau_{\lambda_{0}}\right)=\int \varphi(\lambda) \mathrm{e}^{-\tau_{\lambda_{0}} \varphi(\lambda) / \varphi_{0}} \mathrm{~d} \lambda
$$


is modeled with a Lorentzian spectral line shape, $\varphi(\lambda)$,

$$
\varphi(\lambda)=\frac{1}{\pi} \frac{0.5 \Delta \lambda_{S}}{\left(\lambda-\lambda_{0}\right)^{2}+\left(0.5 \Delta \lambda_{S}\right)^{2}}
$$

where $\Delta \lambda_{\mathrm{s}}$ denotes the full-width at half-maximum (FWHM) of the normalized spectral line shape of magnitude, $\varphi_{0}$, at line center. The plasma optical thickness at line center, $\tau_{\lambda_{0}}$,

$$
\tau_{\lambda_{0}}=\int_{-\ell}^{0} \kappa\left(\lambda_{0}\right) \mathrm{d} \ell
$$

is defined in terms of integrated absorption coefficient, $\kappa(\lambda)$, of a spectral line measured along the line-of-sight, $\ell$, at the transition wavelength, $\lambda_{0}$. Figure 5 illustrates results for $\tau_{\lambda_{0}}$ ranging from 0.25 to 2 at equal steps of 0.25 , and for fixed Lorentzian FWHM of $\Delta \lambda_{\mathrm{s}}=0.5 \mathrm{~nm}$. The line shape indicates a flat top for unity optical thickness, i.e., $\tau_{\lambda_{0}}=1$. For values higher than unity, self-absorption affects the line shape primarily at the center ${ }^{2,3}$.

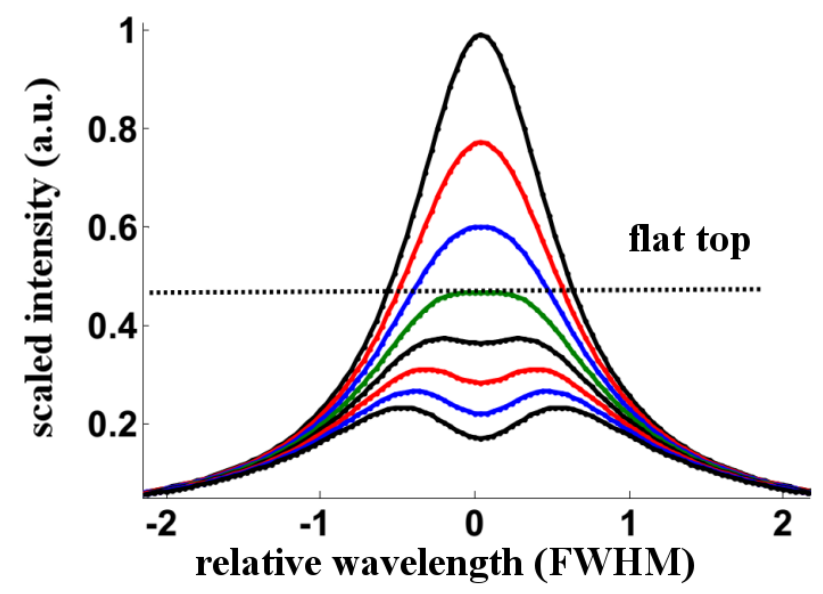

Figure 5: Line shapes $\varphi(\lambda) \mathrm{e}^{-\tau_{\lambda_{0}} \varphi(\lambda) / \varphi_{0}}$ vs. $\lambda$ for fixed $\Delta \lambda_{s}=0.5 \mathrm{~nm}$. Values of $\tau_{\lambda_{0}}$ range from 0.25 to 2.0 in steps of 0.25 .

The fitting of the argument in Eq. (1) to the experimentally measured (distorted) line shape yields two line shape parameters, namely, the distorted Lorentzian FWHM, $\Delta \lambda_{\mathrm{S} 1}$, and the optical depth, $\tau_{\lambda_{0}}$, at line center. The selfreversal parameter, SR,

$$
\mathrm{SR}=\frac{1-\mathrm{e}^{-\tau} \lambda_{0}}{\tau_{\lambda_{0}}} \leq 1
$$

indicates the ratio of transmitted and of weakly $(\kappa(\lambda) \ell<<1)$ affected intensities at line center, or in terms of the transmittance, $\mathrm{T}\left(\tau_{\lambda_{0}}\right)=\mathrm{SR}$. Self-reversal diminishes the peak spectral radiance, and it causes enlarged FWHM, $\Delta \lambda_{\mathrm{S} 2}$, with $\Delta \lambda_{\mathrm{S} 2}>\Delta \lambda_{\mathrm{S} 1}$. Similar to the derivation of self-absorption ${ }^{12}, \Delta \lambda_{\mathrm{S} 2}=\Delta \lambda_{\mathrm{S} 1} \mathrm{SR}^{\alpha}$, or

$$
\mathrm{SR}^{\alpha}=\frac{\Delta \lambda_{\mathrm{S} 2}}{\Delta \lambda_{\mathrm{S} 1}}
$$

The value for the exponent is taken to be $\alpha=-0.54$, in analogy to previously reported self-absorption studies ${ }^{12}$. The self-absorption factor ${ }^{12}$, SA, is functionally identical to that for the self-reversal factor, SR,

$$
\mathrm{SA}^{\alpha}=\frac{\Delta \lambda}{\Delta \lambda_{0}}
$$

Here, $\Delta \lambda$ and $\Delta \lambda_{0}$ indicate the FWHM of spectral lines with and without self-absorption, respectively. 
Figure 6 summarizes a typical example of spectral line shape analysis of the line Ag I lines at 327.9 and $338.2 \mathrm{~nm}$. The figure also shows various stages of line distortion. Figs. (B) and (E) indicate self-absorption, and Figs. (C) and (D) display self-reversal. The electron density, $\mathrm{n}_{\mathrm{e}}$, and the plasma transmission percentages (typically $33 \%$ and $40 \%$ for the reported experiments) are included in the figures. The theoretical, asymptotic form for the transmittance of a Lorentzian line profile ${ }^{3}$ equals

$$
\mathrm{T}_{\text {theory }}\left(\tau_{\mathrm{SR}}\right) \sim 1 / \sqrt{\pi \tau_{\mathrm{SR}}} \text {. }
$$

The theoretical transmittances are compatible with SR factors of 0.32 and 0.38 . The measured line shapes are Lorentzian especially in the wings. However, this discussion neglects Gaussian components from the instrumental broadening of $\Delta \lambda_{\text {instrument }} \sim 0.12 \mathrm{~nm}$. Figs. $6(\mathrm{C})$ and $(\mathrm{F})$ show the effect of self-reversal on line FWHM and spectral intensity. Significant reduction in intensity occurs along with an increase in broadening $\left(\Delta \lambda_{\mathrm{s} 2}\right)$. The self-reversal coefficients are relatively small $(S R=0.32$ and $S R=0.38)$, but due to the effects primarily at the line center ${ }^{3,6,10}$, dips occur as well as a decrease in intensity by one order of magnitude. Noteworthy in this work, self-reversal (quantified by the coefficient SR) is almost independent of the laser fluence, but self-absorption (SA) changes monotonically with laser fluence.

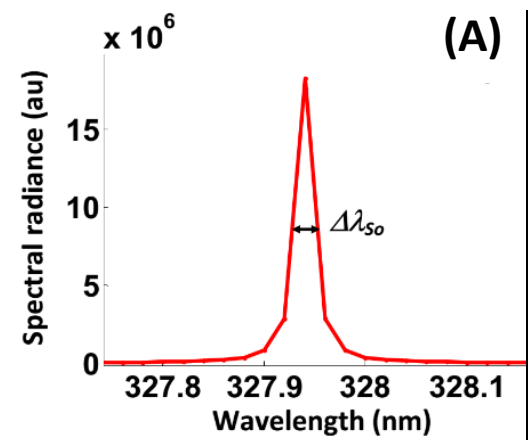

$\Delta \lambda_{\mathrm{So}}=0.017 \mathrm{~nm}, \mathrm{I}_{0}=1.8 \times 10^{7} \mathrm{au}$,
$\mathbf{n}_{\mathrm{e} 0}=3.5 \times 10^{17} \mathrm{~cm}^{-3}, \quad \mathrm{SA}=1$

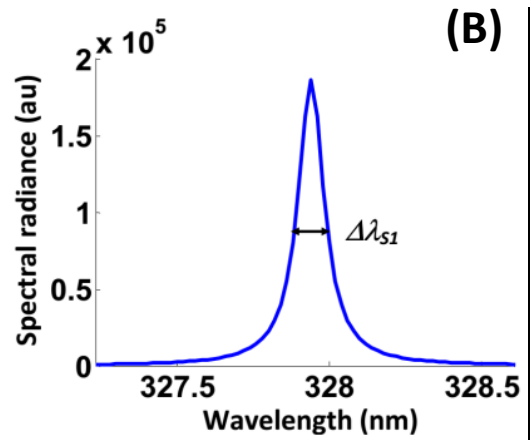

$\Delta \lambda_{\mathrm{s} 1}=0.2 \mathrm{~nm}, \quad \mathrm{I}_{1}=1.8 \times 10^{5}$ au, $\mathrm{n}_{\mathrm{e} 1}=4.2 \times 10^{18} \mathrm{~cm}^{-3}, \quad \mathrm{SA}=0.01$

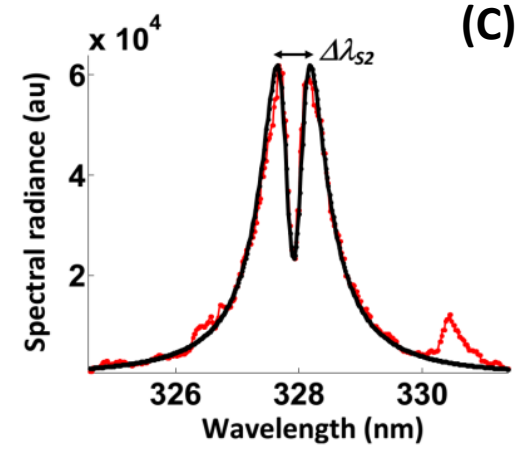

$\Delta \lambda_{\mathrm{s} 2}=0.37 \mathrm{~nm}, \quad I_{2}=6.2 \times 10^{4} \mathrm{au}$, $\mathrm{SR}=0.30, \quad \mathrm{~T}\left(\lambda_{0}\right)=33 \%$

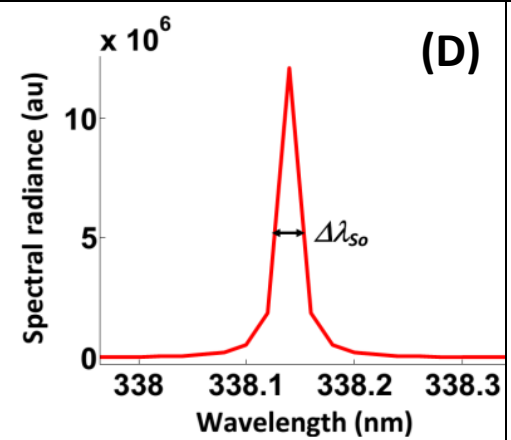

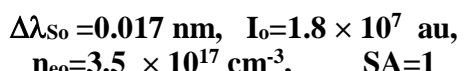

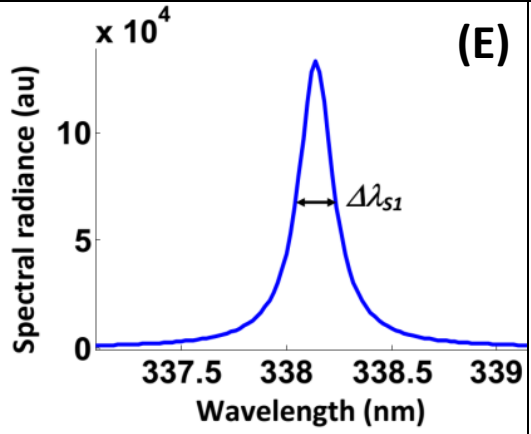

$\Delta \lambda_{\mathrm{s} 1}=0.19 \mathrm{~nm}, \quad \mathrm{I}_{1}=1.3 \times 10^{5} \mathrm{au}$, $n_{\mathrm{e} 1}=3.9 \times 10^{18} \mathrm{~cm}^{-3}, \quad \mathrm{SA}=0.01$

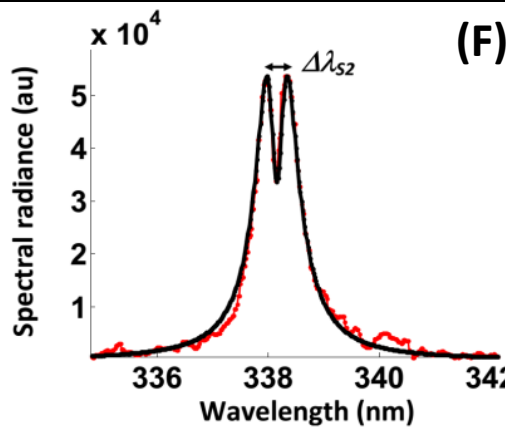

$\Delta \lambda_{\mathrm{s} 2}=0.32 \mathrm{~nm}, I_{2}=5.4 \times 10^{4}$ au, SR=0.39, $\quad \mathbf{T}\left(\lambda_{0}\right)=40 \%$

Figure 6: Analysis for self-reversal (C) and (F), and self-absorption (B) and (E) of the resonance lines at $327.9 \mathrm{~nm}(\mathrm{~A})$ and $338.2 \mathrm{~nm}(\mathrm{~B})$. UV light pulses of fluence $13.5 \mathrm{~J} / \mathrm{cm}^{2}$ at the wavelength of $355 \mathrm{~nm}$ generate the laser plasma.

In this example, the self-reversal peak separation provides values for $\Delta \lambda_{\mathrm{s} 2}$, using the FWHM would cause even larger discrepancies for the electron density, $\mathrm{n}_{\mathrm{e}}$. From Equation (5), computed $\Delta \lambda_{\mathrm{s} 1}$ show $\mathrm{n}_{\mathrm{e}}$ that are $\sim$ ten times higher than the results obtained from an optically thin line. When using lower fluence levels for these two lines, 
larger variances occur in the inferred $n_{e}$ values. From Eq. (6), a factor of ten higher electron density means that the self-absorption factor is of the order of $\mathrm{SA} \sim 0.01$. For self-absorption, the magnitude of the peak spectral irradiances can be evaluated ${ }^{12}$ using $\mathrm{I}_{0}\left(\lambda_{0}\right) \sim \mathrm{I}_{1}\left(\lambda_{0}\right) / \mathrm{SA}$, leading to two orders of magnitude higher irradiances, clearly confirming significant self-absorption and line reversal for the selected example.

\section{CONCLUSIONS}

Self-absorption may lead to a decrease in the peak line intensity of the order of two orders of magnitude, including appearance of self-revered lines. Even after taking into consideration the line shape effects, occurrence of selfabsorption for a particular line contraindicates plasma electron density and temperature measurements from that line. The experimentally measured transmission factors for both lines 327.9 and $338.2 \mathrm{~nm}$ change with incident laser fluence. The theoretical analysis predicts transmittance values consistent with the measured ones within the experimental margins of error. The optically thin silver line at $827 \mathrm{~nm}$ allows one to determine the electron density showing decreases as expected from $3.5 \times 10^{17}$ to $1.1 \times 10^{16} \mathrm{~cm}^{-3}$ with decreasing laser fluence. However, as selfabsorption of the silver $338.2 \mathrm{~nm}$ line decreases with decreasing fluence, the variations of inferred electron densities are larger than anticipated, or the $338.2 \mathrm{~nm}$ line shows a larger standard deviations than that for the 827 $\mathrm{nm}$ line. The Ag I line at $338.2 \mathrm{~nm}$ disappears for a laser fluence of $2.1 \mathrm{~J} / \mathrm{cm}^{2}$. Finally, plasma opacity manifests itself as a combination of self-absorption and self-reversal effects, and line recovery requires results from additional analysis of an optically thin line.

\section{REFERENCES}

[1] C.G. Parigger, D.M. Surmick, G. Gautam, A.M. EL Sherbini, Opt. Lett. 40 (2015) 3436.

[2] C.G. Parigger, D.M. Surmick, G. Gautam, J. Phys.: Conf. Ser. 810 (2017) 012012.

[3] F.E. Irons, J. Quant. Spectrosc. Radiat. Transfer 22 (1979) 1.

[4] T. Holstein, Phys. Rev. 72 (1947) 1212.

[5] T. Holstein, Phys. Rev. 83 (1951) 1159.

[6] R.D. Cowan, G.H. Dieke, Rev. Mod. Phys. 20 (1948) 418.

[7] J.F. Kielkopf, N.F. Allard, J. Phys B: At. Mol. Opt. Phys. 47 (2014) 155701.

[8] H.-Y. Moon, K.K. Herrera, N. Omenetto, B.W. Smith, J.D. Winefordner, Spectrochim. Acta Part B 64 (2009) 702.

[9] A.M. El Sherbini, C.G. Parigger, Spectrochim. Acta Part B 116 (2016) 8.

[10] T. Fujimoto, Plasma Spectroscopy, Clarendon Press, Oxford, GB, 2004.

[11] H.-J. Kunze, Introduction to Plasma Spectroscopy, Springer-Verlag, Berlin, DE, 2006.

[12] A.M. El Sherbini, Th.M. El Sherbini, H. Hegazy, G. Cristoforetti, S. Legnaioli, V. Palleschi, L. Pardini, A. Salvetti, E. Tognoni, Spectrochim. Acta Part B 60 (2005) 1573. 